\title{
Fundamental limitations in the purifications of tensor networks
}

\author{
G. De las Cuevas, ${ }^{1}$ T. S. Cubitt, ${ }^{2}$ J. I. Cirac, ${ }^{1}$ M. M. Wolf, ${ }^{3}$ and D. Pérez-García ${ }^{4}$ \\ ${ }^{1}$ Max Planck Institute for Quantum Optics, \\ Hans-Kopfermann-Str. 1, 85748 Garching, Germany \\ ${ }^{2}$ Department of Computer Science, University College London, London WC1E 6BT, UK, and \\ DAMTP, University of Cambridge, Centre for Mathematical Sciences, \\ Wilberforce Road, Cambridge CB3 OWA, UK \\ ${ }^{3}$ Zentrum Mathematik, Technische Universität München, 85748 Garching, Germany \\ ${ }^{4}$ Departamento de Análisis Matemático and IMI, \\ Universidad Complutense de Madrid, 28040 Madrid, Spain \\ and ICMAT, C/ Nicolás Cabrera, Campus de Cantoblanco, 28049 Madrid, Spain
}

\begin{abstract}
We show a fundamental limitation in the description of quantum many-body mixed states with tensor networks in purification form. Namely, we show that there exist mixed states which can be represented as a translationally invariant (TI) matrix product density operator (MPDO) valid for all system sizes, but for which there does not exist a TI purification valid for all system sizes. The proof is based on an undecidable problem and on the uniqueness of canonical forms of matrix product states. The result also holds for classical states.
\end{abstract}

\section{INTRODUCTION AND MAIN RESULT}

In recent years, Tensor Network States (TNS) have become a major tool in the study of quantum many-body systems. Because of their ability to capture the entanglement structure present in grounds states [1-3], thermal states [4], low energy states within a phase [5], or in a phase transition [6], they constitute a powerful ansatz to describe and simulate strongly correlated quantum systems in an efficient way. They provide an accurate description of the target states with a number of parameters that only grows polynomially with the size of the system, overcoming the exponential blow-up of the total Hilbert space of the system.

Here we will restrict to a particular class of TNS for the description of mixed states in one spatial dimension (1D), the so-called Matrix Product Density Operators (MPDO) [7, 8]. In the classical case (i.e. for states diagonal in the computational basis), they can be written as

$$
\rho_{A}^{L}=\sum_{i_{1} \ldots i_{L}} \operatorname{Tr}\left(A_{i_{1}} \cdots A_{i_{L}}\right)\left|i_{1} \cdots i_{L}\right\rangle\left\langle i_{1} \cdots i_{L}\right|
$$

where $i_{j}=1, \ldots, d$ and $A_{i} \in \mathcal{M}_{D}$, where the latter denotes the set of $D \times D$ complex matrices. MPDOs play a chief role in the study of $2 \mathrm{D}$ systems, since they describe the so-called boundary theory, which encodes the relevant properties of the system. For this reason, they are the objects based on which one classifies the phases and phase transitions in 2D models [9]. They are also relevant in the study of $1 \mathrm{D}$ open quantum systems $[10,11]$, in the very same way as their pure analogues - Matrix Product States (MPS) $[2,3,12]$ - are central in the study of closed 1D systems.

Ever since MPDOs were introduced, a major problem became clear: It was not easy to detect in the local matrices $A_{i}$ whether the global quantum state $\rho_{A}^{L}$ was positive semidefinite, as desired. This still constitutes at one of the difficulties in the contraction of Projected Entangled Pair States (PEPS) in 2 dimensions [13-15], and in the simulation of 1D open systems.

In order to circumvent this problem, a particular type of MPDO was introduced [7], in which positivity was guaranteed by construction: the so-called purification form, given by a MPS with a local environment at each site,

$$
|\Psi\rangle=\sum_{i, e} \operatorname{Tr}\left(B_{i_{1}, e_{1}} B_{i_{2}, e_{2}} \cdots B_{i_{L}, e_{L}}\right)\left|i_{1} e_{1} i_{2} e_{2} \cdots i_{L} e_{L}\right\rangle
$$

where $B_{i, e} \in \mathcal{M}_{\tilde{D}}$. The resulting (unnormalized) mixed state emerges by tracing all the local environments:

$$
\sigma_{B}^{L}=\operatorname{Tr}_{e_{1}, \ldots e_{L}}|\Psi\rangle\langle\Psi| .
$$

Note that in this case $\sigma_{B}^{L} \geq 0$ [22] for all $L$ by construction, whereas $\rho_{A}^{L} \geq 0$ for all $L$ by assumption.

It is not difficult to see that, for fixed $L$ and matrices $A_{i}$, one can always find matrices $B_{i, e}$ of finite size $\tilde{D}$ for which $\rho_{A}^{L} / \operatorname{Tr}\left(\rho_{A}^{L}\right)=\sigma_{B}^{L} / \operatorname{Tr}\left(\sigma_{B}^{L}\right)$. What is not clear is whether this can be done simultaneously for all $L$. In this work we will show that, in general, this cannot be done: 
Theorem 1 (Main result). There exist MPDOs $\left\{\rho_{A}^{L}\right\}_{L}$ given by matrices $\left(A_{i}\right)$ (meaning that $\rho_{A}^{L} \geq 0$ for all $L)$ for which there does not exist another MPDO in purification form $\left\{\sigma_{B}^{L}\right\}_{L}$ (see Eq. (2)) such that

$$
\frac{\rho_{A}^{L}}{\operatorname{Tr}\left(\rho_{A}^{L}\right)}=\frac{\sigma_{B}^{L}}{\operatorname{Tr}\left(\sigma_{B}^{L}\right)} \quad \forall L .
$$

The result holds true even if we restrict ourselves to $\left\{A_{i}\right\}_{i=1}^{7}$, all $A_{i}$ rational-valued $7 \times 7$ matrices, and the state $\rho_{A}^{L}$ a classical state (i.e. diagonal in the computational basis) for all $L$.

The proof technique is somehow non-standard since it relies on the notion of undecidability. In Section II we will show that the property

$$
\text { (P) } \quad \rho_{A}^{L} \geq 0 \quad \forall L
$$

is undecidable, in the same sense as the halting problem of a Turing machine. The fact that this type of problems can be undecidable was recently observed in [16], where the analogue problem for open boundary conditions is considered. Note that separations between MPDOs and their purification form were given in [17], but in the non translationally invariant case. In Section III we will prove some results about the canonical forms of MPS, which essentially show that if a purification $\left\{\sigma_{B}^{L}\right\}_{L}$ satisfying (3) exists, then it can be found in a finite amount of time. Finally, we gather all results in Section IV to prove Theorem 1. We conclude and give some outlook in Section V.

\section{UNDECIDABILITY OF THE POSITIVITY PROBLEM}

We consider the following problem related to positivity of density matrices.

Problem 2. Given $\left\{A_{i} \in \mathbb{Z}^{7 \times 7}\right\}_{i=1}^{7}$, one is promised that either

Case 1. $\rho_{A}^{L} \geq 0$ for all $L$; or

Case 2. There exists $L_{0}$ such that $\rho_{A}^{L} \nsupseteq 0$ for all $L \geq L_{0}$.

Decide which case holds.

Lemma 3. Problem 2 is undecidable.

Proof. The proof of Lemma 3 relies on the recent proof that the Zero in the Upper Left Corner (ZULC) problem is undecidable for 5 integer matrices of size $3 \times 3$ [18]. (In [18] they consider rational-valued matrices, but by multiplying all of them by the least common multiple of all denominators involved, one trivially obtains the result for integer-valued matrices.)

In such ZULC problem, one is given five $3 \times 3$ matrices $Y_{1}, \ldots Y_{5}$ and one is asked to decide whether there exists an $N \in \mathbb{N}$ and a sequence $i_{1}, \ldots i_{N} \in\{1, \ldots 5\}$ so that

$$
\left\langle 0\left|Y_{i_{1}} \cdots Y_{i_{N}}\right| 0\right\rangle=0 .
$$

In Ref. [18] it was shown that this problem is undecidable even with the additional promise that $Y_{1}, \ldots Y_{5}$ can be jointy upper-triangularized, that is, there exists a non-singular matrix $Q$ so that, all $Q Y_{i} Q^{-1}$ are upper-triangular. In the following we reduce this problem to Problem 2.

Consider the projector onto the 6 -dimensional symmetric subspace $P:=\frac{1}{2}(\mathbb{1}+\mathbb{F})$, where $\mathbb{1}$ is the identity matrix of size $3 \times 3$, and $\mathbb{F}$ the 'flip' operator,

$$
\mathbb{F}=\sum_{i, j=0,1,2}|i, j\rangle\langle j, i| .
$$

Let $\mathcal{O}$ be a real isometry of size $6 \times 9$ such that $\mathcal{O O}^{\dagger}=\mathbb{1}($ of size $6 \times 6)$ and $\mathcal{O}^{\dagger} \mathcal{O}=P$. We define

$$
A_{i}=\mathcal{O} P\left(Y_{i} \otimes Y_{i}\right) P \mathcal{O}^{\dagger} \oplus|6\rangle\langle 6|, \quad \text { for } i=1, \ldots, 5,
$$

where $\mathcal{O} P\left(Y_{i} \otimes Y_{i}\right) P \mathcal{O}^{\dagger}$ acts on $\mathbb{C}^{6}=\operatorname{span}\{|0\rangle, \ldots,|5\rangle\}$. We also define

$$
\begin{aligned}
& A_{6}=(|0\rangle\langle 0|\otimes| 0\rangle\langle 0|) \oplus|6\rangle\langle 6|, \\
& A_{7}=(|0\rangle\langle 0|\otimes| 0\rangle\langle 0|) \oplus(-|6\rangle\langle 6|) .
\end{aligned}
$$


Note that $A_{i} \in \mathbb{Q}^{7 \times 7}$ for all $i$.

We claim that there is a word $j_{1}, \ldots, j_{m}$ such that

$$
\left\langle 0\left|Y_{j_{1}} Y_{j_{2}} \ldots Y_{j_{m}}\right| 0\right\rangle=0
$$

if and only if $\left\{A_{i}\right\}$ (as defined in (6),(7),(8)) is in Case 2 of Problem 2. Our proof will also show that $\left\{A_{i}\right\}$ satisfies the promise of Problem 2 (i.e. they belong either to Case 1 or 2 ).

For the "only if" direction, take the word $\left(j_{1}, \ldots, j_{m}\right)$ that satisfies $(9)$, and define $\left(i_{1}, \ldots, i_{L_{0}}\right)=$ $\left(j_{1}, \ldots, j_{m}, 7\right)$. Using that $P \mathcal{O}^{\dagger}|0,0\rangle=|0,0\rangle$, that $P$ commutes with $Y_{i} \otimes Y_{i}$, and basic properties of the trace, we obtain that

$$
\operatorname{Tr}\left(A_{i_{1}} \ldots A_{i_{L_{0}}}\right)=\left(\left\langle 0\left|Y_{j_{1}} \ldots Y_{j_{m}}\right| 0\right\rangle\right)^{2}-1
$$

which is negative. Moreover, appending an arbitrary number of 6 at the end of $i_{1}, \ldots, i_{L_{0}}$ gives words of arbitrary length $L \geq L_{0}$ with a negative trace as in (10). Thus $\rho_{A}^{L} \ngtr 0$ for all $L \geq L_{0}$.

Conversely, assume that there is a word $i_{1}, \ldots, i_{L}$ (for some $L \geq L_{0}$ ) such that $\operatorname{Tr}\left(A_{i_{1}} \ldots A_{i_{L}}\right)<0$. Assume first that this word contains no 6 or 7 . Using the above-mentioned properties of $\mathcal{O}$ and $P$, and that $\operatorname{Tr}(\mathbb{F}(G \otimes G))=\operatorname{Tr}\left(G^{2}\right)$ for any matrix $G$, we obtain

$$
\operatorname{Tr}\left(A_{i_{1}} \ldots A_{i_{L}}\right)=\frac{1}{2}\left[\left(\operatorname{Tr}\left(Y_{i_{1}} \ldots Y_{i_{L}}\right)\right)^{2}+\operatorname{Tr}\left(\left(Y_{i_{1}} \ldots Y_{i_{L}}\right)^{2}\right)\right]+1 .
$$

The first term in square brackets is clearly non-negative. The second is non-negative because, as mentioned above, we can assume without loss of generality that the matrices $Y_{i}$ can be simultaneously put in upper triangular form. Thus the word must contain at least a 6 or a 7 .

If it only contains one 6 , imagine it is in the last position of the word (which can always be achieved by using cyclicity of the trace). We obtain

$$
\operatorname{Tr}\left(A_{i_{1}} \ldots A_{i_{L}}\right)=\left(\left\langle 0\left|Y_{i_{1}} \ldots Y_{i_{L-1}}\right| 0\right\rangle\right)^{2}+1
$$

which is always positive. An analogous argument holds if there are multiple $6 \mathrm{~s}$.

Hence the word must contain at least one 7, and imagine again that there is just one 7 and this is the last element of the word. In this case,

$$
\operatorname{Tr}\left(A_{i_{1}} \ldots A_{i_{L}}\right)=\left(\left\langle 0\left|Y_{i_{1}} \ldots Y_{i_{L-1}}\right| 0\right\rangle\right)^{2}-1
$$

which is negative by assumption. Since the $Y_{i}$ 's contain only integer numbers, this implies that $\left\langle 0\left|Y_{i_{1}} \ldots Y_{i_{L-1}}\right| 0\right\rangle=0$, as we wanted to show. An analogous argument holds if there are multiple $7 \mathrm{~s}$.

As commented above, by multiplying all matrices $A_{i}$ by the least common multiple of all denominators involved in them, one can assume without loss of generality that they have integer coefficients instead of rational ones.

\section{CANONICAL FORMS OF MATRIX PRODUCT STATES}

In this Section we will show a result about canonical forms of MPS. This will allows us to argue in Section IV that determining whether there exists a purification that satisfies (3) is decidable. The following results are based on Ref. [9], but here we will give the explicit bounds. Since we will only consider MPDOs diagonal in the computational basis, we will consider them as MPS by mapping $|i\rangle\langle i|$ to $|i\rangle$. That is, the pure state corresponding to

$$
\rho_{A}^{L}=\sum_{i_{1} \ldots i_{L}} \operatorname{Tr}\left(A_{i_{1}} \cdots A_{i_{L}}\right)\left|i_{1} \cdots i_{L}\right\rangle\left\langle i_{1} \cdots i_{L}\right|
$$

is

$$
\left|\psi_{A}^{L}\right\rangle=\sum_{i_{1} \ldots i_{N}} \operatorname{Tr}\left(A_{i_{1}} \cdots A_{i_{L}}\right)\left|i_{1} \cdots i_{L}\right\rangle
$$

We also denote by $\left|\psi_{C}^{L}\right\rangle$ the pure state corresponding to the MPDO in purification form associated to $\left\{B_{i, e} \in \mathcal{M}_{\tilde{D}}\right\}$ (see Fig. 1). More precisely,

$$
\left|\psi_{C}^{L}\right\rangle=\sum_{i_{1} \ldots i_{N}} \operatorname{Tr}\left(C_{i_{1}} \cdots C_{i_{L}}\right)\left|i_{1} \cdots i_{L}\right\rangle
$$


(a)

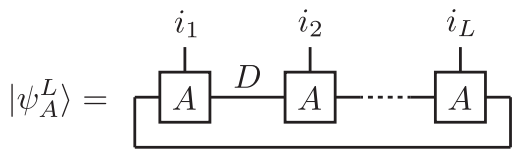

(b)

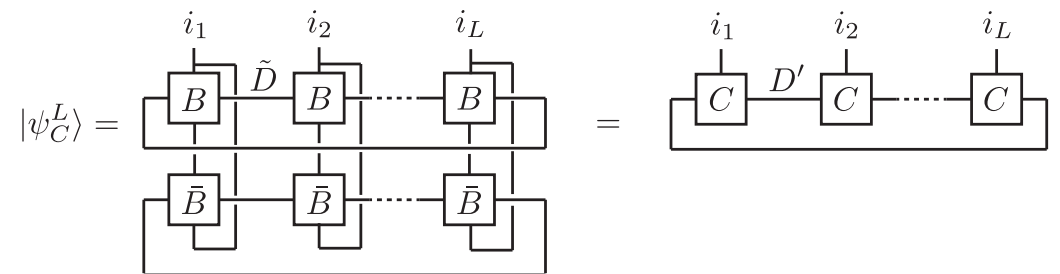

(c)

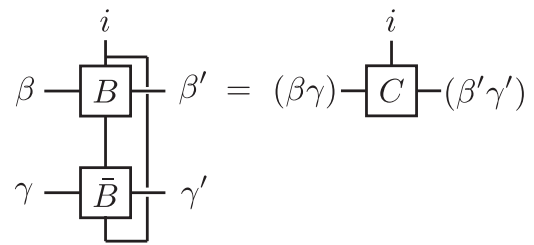

FIG. 1: The tensor network diagrams for (a) $\left|\psi_{A}^{L}\right\rangle$ (see Eq. (14)), (b) $\left|\psi_{C}^{L}\right\rangle$ (see Eq. (15)) and (c) $C$ (see Eq. (16)).

with

$$
\left(C_{i}\right)_{(\beta, \gamma),\left(\beta^{\prime}, \gamma^{\prime}\right)}=\sum_{k}\left(B_{i, k}\right)_{\beta, \beta^{\prime}}\left(\bar{B}_{i, k}\right)_{\gamma, \gamma^{\prime}}
$$

Note that the size of the matrices $C_{i}$ is $D^{\prime}=\tilde{D}^{2}$.

The main result that we need concerning MPS is the following. We will state the results for general matrices $\left\{A_{i}\right\},\left\{C_{i}\right\}$ but finally we will apply them to $C_{i}$ of the form (16).

Theorem 4. Let $\left\{A_{i} \in \mathcal{M}_{D}\right\}_{i=1}^{d},\left\{C_{i} \in \mathcal{M}_{D^{\prime}}\right\}_{i=1}^{d}$ be two families of matrices for which there exist constants $m_{L}$ such that

$$
\left|\psi_{A}^{L}\right\rangle=m_{L}\left|\psi_{C}^{L}\right\rangle \quad \forall L \leq D ! D^{\prime} ! 3\left(D+D^{\prime}\right)^{6} .
$$

Then there exist $m_{L}$ such that $\left|\psi_{A}^{L}\right\rangle=m_{L}\left|\psi_{C}^{L}\right\rangle$ for all $L$ which are multiples of $D ! D^{\prime} ! 3\left(D+D^{\prime}\right)^{5}$.

To prove Theorem 4, we need to gather some facts about MPS. We start with the canonical forms of MPS.

Theorem 5 (Canonical form (Theorem 4 in [19])). Given $\left\{\tilde{A}_{i} \in \mathcal{M}_{\tilde{D}}\right\}_{i=1}^{d}$, there exists another set of matrices $\left\{A_{i} \in \mathcal{M}_{D}\right\}_{i=1}^{d}$ with $D \leq \tilde{D}$ so that $\left|\psi_{A}^{L}\right\rangle=\left|\psi_{\tilde{A}}^{L}\right\rangle$ for all $L$ and the $A_{i}$ are block diagonal, $A_{i}=\oplus_{j} \lambda_{j} A_{i}^{j}$, and satisfy that for all $j$

(i) $\lambda_{j}>0$,

(ii) $\mathbb{1}$ is the only fixed point of the operator $\mathcal{E}(X)=\sum_{i} A_{i}^{j} X A_{i}^{j \dagger}$, and

(iii) the unique fixed point of its adjoint map $\mathcal{E}^{*}(Y)=\sum_{i} A_{i}^{j \dagger} Y A_{i}^{j}$ is a full rank positive diagonal matrix $\Lambda_{j}$ (normalized so that $\operatorname{Tr}\left(\Lambda_{j}\right)=1$ ).

The blocks in the decomposition $A_{i}=\oplus_{j} \lambda_{j} A_{i}^{j}$ may not be injective yet. To make them injective, we only need to group $D$ ! sites together, since the periodicity of each block is a divisor of the size of the block (see Lemma 5 of [20], [12]).

Theorem 6 (Quantum Wielandt [21]). Consider the matrices $\left\{A_{i} \in \mathcal{M}_{D}\right\}_{i=1}^{d}$, so that the corresponding $M P S$ becomes injective after blocking $L_{0}$ sites. Then $L_{0}$ can be taken to be $D^{4}-1$.

Now we show that if two injective MPS are proportional for some lengths, then their matrices must be related by a unitary. 
Theorem 7 (Injective case). Assume that $\left\{A_{i} \in \mathcal{M}_{D}\right\}$ and $\left\{C_{i} \in \mathcal{M}_{D^{\prime}}\right\}$ are such that $\left|\psi_{A}^{L}\right\rangle$ and $\left|\psi_{C}^{L}\right\rangle$ become injective at some length $L_{0}=D^{4}-1$, and both verify (ii) and (iii) of Theorem 5. Assume that $D^{\prime} \leq D$. Fix $L$ so that $L \geq 2 L_{0}+1$ and assume that $\left|\psi_{A}^{L}\right\rangle$ is proportional to $\left|\psi_{C}^{L}\right\rangle$. Then $D=D^{\prime}$ and there exists a unitary $U$ and a phase $e^{\mathrm{i} \phi}$ so that $A_{i}=e^{\mathrm{i} \phi} U C_{i} U^{\dagger}$ for all $i$.

Proof. Let $h$ denote the interaction hamiltonian of range $L_{0}+1$ that has $\left|\psi_{A}^{L}\right\rangle$ as its unique ground state (up to constant) in any chain of length $N \geq 2 L_{0}+1$ (Theorem 10 of [19] and Theorem 6 for the injectivity bound of $\left.\left\{A_{i}\right\}\right)$. Using injectivity of $\left\{\bar{C}_{i}\right\}$ and the hypothesis it is easy to see that $\left|\psi_{C}^{L}\right\rangle$ is also a ground state for $h$ for all chains of length $L \geq 2 L_{0}+1$. Therefore $\left|\psi_{C}^{L}\right\rangle$ and $\left|\psi_{A}^{L}\right\rangle$ are proportional for all $L \geq 2 L_{0}+1$. Finally we use Lemma 3 of [20] to conclude the proof.

Now we state that each of the injective blocks is linearly independent.

Theorem 8 (Linear independence of injective blocks (Proposition 4 of [19])). Let $\left\{A_{i}^{1}\right\}, \ldots,\left\{A_{i}^{b}\right\}$ be of sizes $D_{1} \geq \cdots \geq D_{b}$, so that all of them define injective MPS, each of them is in canonical form, and assume that neither of them is related by $e^{\mathrm{i} \phi} U \cdot U^{\dagger}$. Then for all $L \geq 3(b-1) D_{1}^{4}$ the vectors $\left|\psi_{A^{1}}^{L}\right\rangle, \ldots,\left|\psi_{A^{b}}^{L}\right\rangle$ are linearly independent.

Finally we need to collect some facts about moments of sets of numbers.

Lemma 9. Let $\alpha_{1}, \ldots, \alpha_{r}, \beta_{1}, \ldots, \beta_{n} \in \mathbb{C}$ with $n \geq r$ so that

$$
\sum_{i=1}^{r} \alpha_{i}^{L}=\sum_{j=1}^{n} \beta_{j}^{L}, \quad \text { for all } L=1, \ldots, n .
$$

Complete them as $\alpha_{r+1}=\cdots=\alpha_{n}=0$. Then there exists a permutation $P \in \Pi_{n}$ so that

$$
\left(\alpha_{1}, \ldots, \alpha_{n}\right)=\left(\beta_{P(1)}, \ldots, \beta_{P(n)}\right) .
$$

This result is standard but we include the proof for completeness.

Proof. Let $s_{k}$ denote the power sum polynomials in $n$ variables, $s_{k}=x_{1}^{k}+\cdots+x_{n}^{k}$, and

$$
\tau_{k}=\sum_{i_{1}<i_{2}<\cdots<i_{k}} x_{i_{1}} x_{i_{2}} \cdots x_{i_{k}} .
$$

We have the following Newton identities:

$$
\begin{aligned}
\tau_{1} & =s_{1}, \\
\tau_{k} & =(-1)^{k-1} \frac{1}{k}\left(s_{k}-\tau_{1} s_{k-1}+\cdots+(-1)^{k-1} \tau_{k-1} s_{1}\right),
\end{aligned}
$$

which allow us to write all $\tau_{1}, \ldots, \tau_{n}$ as polynomials on $s_{1}, \ldots, s_{n}$.

Now we consider a new variable $X$ and

$$
f(X)=\left(X-x_{1}\right)\left(X-x_{2}\right) \cdots\left(X-x_{n}\right)
$$

By Vietá's formula, we have

$$
f=X^{n}-\tau_{1} X^{n-1}+\tau_{2} X^{n-2}+\cdots+(-1)^{n} \tau_{n} .
$$

We define now $f_{\alpha}$ as $f$ with $x_{i}=\alpha_{i}$ and similarly $f_{\beta}$. By the above and the hypothesis, both are polynomials in $X$ with the same coefficients (hence the same polynomial) but one has roots $\alpha_{1}, \ldots, \alpha_{n}$ (with the repetitions given by the multiplicities) and the other $\beta_{1}, \ldots, \beta_{n}$. The conclusion follows.

A trivial corollary of this lemma is the following.

Corollary 10. Let $\alpha_{1}, \ldots \alpha_{r} \in \mathbb{C} \backslash\{0\}$. Then there exists $L \leq r$ so that $\sum_{i=1}^{r} \alpha_{i}^{L} \neq 0$.

Another corollary for the proportional case is the following. 
Corollary 11. For each $\alpha \in\{1, \ldots, \Gamma\}$, consider the vectors $\left(\mu_{\alpha, 1}, \ldots, \mu_{\alpha, r_{\alpha}}\right)$ and $\left(\nu_{\alpha, 1}, \ldots, \nu_{\alpha, r_{\alpha}}\right)$ (if they do not have the same length, we complete them with zeros), and let $L_{0}=\sum_{\alpha} r_{\alpha}$. Assume that for each $L \leq L_{0}^{2}$ there exists a constant $m_{L} \neq 0$ independent of $\alpha$ such that

$$
\sum_{i=1}^{r_{\alpha}} \mu_{\alpha, i}^{L}=m_{L} \sum_{i=1}^{r_{\alpha}} \nu_{\alpha, i}^{L} \quad \forall \alpha
$$

Then for all $L \in \mathbb{N}$ there exists a $m_{L}$ so that (24) holds.

Proof. Since $m_{L} \neq 0$, Eq. (24) is equivalent to

$$
\sum_{i=1}^{r_{\alpha}} \mu_{\alpha, i}^{L} \sum_{j=1}^{r_{\beta}} \nu_{\beta, j}^{L}=\sum_{i=1}^{r_{\alpha}} \nu_{\alpha, i}^{L} \sum_{j=1}^{r_{\beta}} \mu_{\beta, j}^{L} \quad \forall \alpha, \beta
$$

Since this holds for all $L \leq L_{0}^{2}$ and $L_{0}^{2} \geq r_{\alpha} r_{\beta}$, Lemma 9 ensures that the vectors $\left(\mu_{\alpha, i} \nu_{\beta, j}\right)_{i j}$ and $\left(\nu_{\alpha, i} \mu_{\beta, j}\right)_{i j}$ are related by a permutation, which in turn implies that Eq. (25) holds for all $L \in \mathbb{N}$, and thus Eq. (24) also holds for all $L \in \mathbb{N}$.

We are now ready to prove Theorem 4.

Proof of Theorem 4. Consider $\left\{A_{i} \in \mathcal{M}_{D}\right\},\left\{C_{i} \in \mathcal{M}_{D^{\prime}}\right\}$ that satisfy (17). First we block sites into groups of size $D ! D^{\prime}$ ! so that we can assume without loss of generality that both $\left|\psi_{A}\right\rangle$ and $\left|\psi_{C}\right\rangle$ are in canonical form with each block being injective. From now on we will work with the grouped sites so the real system size $L^{\prime}$ will be of the form $L^{\prime}=D ! D^{\prime} ! L$ for some $L$. In the following we will consider just such $L$ 's.

To each vector $\left|\psi_{A}^{L}\right\rangle$ and $\left|\psi_{C}^{L}\right\rangle$ we apply $e^{\mathrm{i} \phi} U \cdot U$ if needed to the different blocks so that those related by such relation become the same (and the phase $e^{\mathrm{i} \phi}$ is absorbed in the $\lambda$ 's, which now become complex numbers). Thus, by applying $V A_{i} V^{\dagger}$ and $W C_{i} W^{\dagger}$ with $V, W$ unitaries we can assume without loss of generality that the $A$ 's and $C$ 's take the form

$$
\begin{gathered}
A_{i}=\oplus_{j \in J}\left(A_{i}^{j} \otimes \operatorname{diag}\left(\lambda_{1}^{j}, \ldots, \lambda_{r_{j}}^{j}\right)\right) \\
C_{i}=\oplus_{k \in K}\left(C_{i}^{k} \otimes \operatorname{diag}\left(\mu_{1}^{k}, \ldots, \mu_{s_{k}}^{k}\right)\right),
\end{gathered}
$$

with $\lambda$ 's and $\mu$ 's complex numbers, and neither of the $A_{i}^{j}$ 's are related to each other by $e^{\mathrm{i} \phi} U \cdot U^{\dagger}$, and the same holds for the $C$ 's.

Define the set $K^{\prime} \subset K$ as follows: $k \in K^{\prime}$ if there exist $j \in J$, a phase $\phi$ and a unitary $U$ so that $e^{\mathrm{i} \phi} U A_{i}^{j} U^{\dagger}=C_{i}^{k}$ for all $i$. We want to see that $K^{\prime}=K$. If this were not the case, by Theorem 8 applied to $\left\{A_{i}^{j}\right\}_{i}$ with $j \in J$ and $\left\{C_{i}^{k}\right\}_{i}$ with $k \in K \backslash K^{\prime}$, we would obtain that for all $L \geq 3\left(D+D^{\prime}\right)^{5}$ the set of vectors $\left|\psi_{A^{j}}^{L}\right\rangle,\left|\psi_{C^{k}}^{L}\right\rangle$ with $j \in J, k \in K \backslash K^{\prime}$ is linearly independent. On the other hand, by the hypothesis (Eq. (17)),

$$
\sum_{j}\left(\sum_{l}\left(\lambda_{l}^{j}\right)^{L}\right)\left|\psi_{A^{j}}^{L}\right\rangle=m_{L} \sum_{k}\left(\sum_{m}\left(\mu_{m}^{k}\right)^{L}\right)\left|\psi_{C^{k}}^{L}\right\rangle .
$$

Thus, to obtain a contradiction, we only need to show that for some $k \notin K^{\prime}$ there exists an $L$ with $3\left(D+D^{\prime}\right)^{5} \leq L \leq 3\left(D+D^{\prime}\right)^{6}$ so that $\sum_{m}\left(\mu_{m}^{k}\right)^{L} \neq 0$. This is true by Corollary 10 applied to $\alpha_{m}=$ $\left(\mu_{m}^{k}\right)^{3\left(D+D^{\prime}\right)^{5}}$. So $K=K^{\prime}$.

Interchanging the roles of $A$ and $C$ one obtains that $K=J$, and, again by applying $V A_{i} V^{\dagger}$ and $W C_{i} W^{\dagger}$ with $V, W$ unitaries, we obtain the form

$$
\begin{aligned}
& A_{i}=\oplus_{j \in J}\left(A_{i}^{j} \otimes \operatorname{diag}\left(\lambda_{1}^{j}, \ldots, \lambda_{r_{j}}^{j}\right)\right) \\
& C_{i}=\oplus_{j \in J}\left(A_{i}^{j} \otimes \operatorname{diag}\left(\mu_{1}^{j}, \ldots, \mu_{s_{j}}^{j}\right)\right) .
\end{aligned}
$$

Now from the linear independence of the vectors $\left|\psi_{A^{j}}^{L}\right\rangle$ (Theorem 8), and the hypothesis (Eq. (17)), it follows that for all $j$,

$$
\sum_{l}\left(\lambda_{l}^{j}\right)^{L}=m_{L} \sum_{m}\left(\mu_{m}^{j}\right)^{L}
$$


for all $3\left(D+D^{\prime}\right)^{5} \leq L \leq 3\left(D+D^{\prime}\right)^{6}$. Applying Corollary 11 to $\alpha_{l}=\left(\lambda_{m}^{l}\right)^{3\left(D+D^{\prime}\right)^{5}}$ and $\beta_{m}=\left(\mu_{m}^{k}\right)^{3\left(D+D^{\prime}\right)^{5}}$ we obtain that $\left|\psi_{A}^{\bar{L}}\right\rangle \propto\left|\psi_{C}^{L}\right\rangle$ for all $L$ 's which are multiples of $3\left(D+D^{\prime}\right)^{5}$. Recalling that the total system size is $L^{\prime}=D ! D^{\prime} ! L$, we obtain the result.

\section{PROOF OF THE MAIN RESULT (THEOREM 1)}

We are finally in a position to prove the main result of this paper.

Proof of Theorem 1. Assume that Theorem 1 is not true, that is, for every tensor $A$ such that $\rho_{A}^{L} \geq 0$ for all $L$, there exists a tensor $B$ of finite size so that $\rho_{A}^{L} \propto \sigma_{B}^{L}$ for all $L$. We will use this fact to design an algorithm that solves an undecidable problem.

So take a family of matrices $\left\{A_{i}\right\}$ which are a valid input to Problem 2 (that is, either Case 1 or Case 2 holds). Then consider the following algorithm:

$D^{\prime}=1, L=1$

while not halt do

Decide if there exists $\left|\psi_{C}^{L}\right\rangle$ with $C_{i}$ of form (16) and size $D^{\prime}$ that verifies Eq. (17).

if it exists, output 'Case 1' and halt.

else diagonalize $\rho_{A}^{L}$.

if $\operatorname{mineig}\left(\rho_{A}^{L}\right)<0$ output 'Case 2' and halt.

else $D^{\prime}=D^{\prime}+1, L=L+1$

end while

Now we show that this algorithm always halts and correctly decides whether $\left\{A_{i}\right\}$ verifies Case 1 or 2 . If $\left\{A_{i}\right\}$ is in Case 2, there exists a finite $L_{0}$ so that $\rho_{A}^{L} \geq 0$ for all $L \geq L_{0}$. Therefore, after $L_{0}$ iterations the algorithm will find it and halt.

If $\left\{A_{i}\right\}$ is in Case 1 , since we are assuming that Theorem 1 is not true, there exists an MPDO in purification form with matrices $\left\{B_{i, e} \in \mathcal{M}_{\sqrt{D^{\prime}}}\right\}$ for some finite $D^{\prime}$ so that (3) holds. By virtue of Theorem 4, deciding whether such $\left\{B_{i, e}\right\}$ exist is a decidable problem, since it consists of deciding whether a system of finitely many polynomial equations (whose unknowns are the entries of $B$ ) has a solution over the complex numbers. Thus, the algorithm will check it and halt after $D^{\prime}$ iterations.

Finding an algorithm to solve Problem 2 shows that it is a decidable problem, which contradicts Lemma 3 and finishes, by contradiction, the proof of Theorem 1.

\section{CONCLUSIONS AND OUTLOOK}

In conclusion, we have shown that there exist mixed states with a TI MPDO description valid for all system sizes, but for which there does not exist a purification which is also translational invariant and valid for all system sizes. To prove this result we have relied on the notion of undecidability and on the uniqueness of canonical forms of MPS. It is an interesting open question for further investigation whether with this result or with similar techniques, one can attack the old question about the existence of Finitely Correlated States which are not $\mathrm{C}^{*}$ Finitely Correlated [12].

\section{Acknowledgements}

GDLC and JIC acknowledge support from SIQS. TSC is supported by the Royal Society. MMW acknowledges support from the CHIST- ERA/BMBF project CQC. The opinions expressed in this publication are those of the authors and do not necessarily reflect the views of the John Templeton Foundation. DPG acknowledges support from MINECO (grant MTM2014-54240-P and ICMAT Severo Ochoa project 
SEV-2015-0554) and Comunidad de Madrid (grant QUITEMAD+-CM, ref. S2013/ICE-2801). This work was made possible through the support of grant \#48322 from the John Templeton Foundation. This project has received funding from the European Research Council (ERC) under the European Union's Horizon 2020 research and innovation programme (grant agreement No 648913).

[1] M. Hastings, Phys. Rev. B 76, 035114 (2007).

[2] U. Schollwöck, Annals of Physics 326, 96 (2011).

[3] F. Verstraete, V. Murg, and J. I. Cirac, Adv. Phys. 57, 143 (2008).

[4] A. Molnár, N. Schuch, F. Verstraete, and J. I. Cirac, Phys. Rev. B 91, 045138 (2014).

[5] J. Haegeman, S. Michalakis, B. Nachtergaele, T. J. Osborne, N. Schuch, and F. Verstraete, Phys. Rev. Lett. 111, 080401 (2013).

[6] G. Vidal, Phys. Rev. Lett. 99, 220405 (2007).

[7] F. Verstraete, J. Garcia-Ripoll, and J. I. Cirac, Phys. Rev. Lett. 93, 207204 (2004).

[8] M. Zwolak and G. Vidal, Phys. Rev. Lett. 93, 207205 (2004).

[9] J. I. Cirac, D. Perez-Garcia, N. Schuch, and F. Verstraete, arxiv:1606.00608 (2016).

[10] T. Prosen and M. Znidaric, Journal of Statistical Mechanics: Theory and Experiment p. P02305 (2008).

[11] J. Cui, J. I. Cirac, and M. C. Bañuls, Phys. Rev. Lett. 114, 220601 (2015).

[12] M. Fannes, B. Nachtergaele, and R. F. Werner, Commun. Math. Phys. 144, 443 (1992).

[13] M. Lubasch, J. I. Cirac, and M. C. Bañuls, New J. Phys. 16, 033014 (2014).

[14] M. Lubasch, J. I. Cirac, and M.-C. Bañuls, Phys. Rev. B 90, 064425 (2014).

[15] A. H. Werner, D. Jaschke, P. Silvi, T. Calarco, J. Eisert, and S. Montangero, Phys. Rev. Lett. 116, 23720 (2016).

[16] M. Kliesch, D. Gross, and J. Eisert, Phys. Rev. Lett. 113, 160503 (2014).

[17] G. De las Cuevas, N. Schuch, D. Perez-Garcia, and J. I. Cirac, New J. Phys. 15, 123021 (2013).

[18] J. Cassaigne, V. Halava, T. Harju, and F. Nicolas, pp. 1-24 (2014), arXiv:1404.0644v2.

[19] D. Perez-Garcia, F. Verstraete, M. M. Wolf, and J. I. Cirac, Quantum Inf. Comput. 7, 401 (2007).

[20] A. Cadarso, M. Sanz, M. M. Wolf, J. I. Cirac, and D. Perez-Garcia, Phys. Rev. B 87, 035114 (2013).

[21] M. Sanz, D. Pérez-García, M. M. Wolf, and J. I. Cirac, IEEE Transactions on Information Theory 56, 4668 (2010).

[22] $A \geq 0$ denotes that $A$ is hermitian and positive semi-definite. 